\title{
Paroxysmal and cognitive phenotypes in Prrt2 mutant mice
}

Louise Robertson ${ }^{1}$, Travis Featherby ${ }^{3}$, Stuart Howell ${ }^{4}$, James Hughes ${ }^{1}$ and Paul Thomas ${ }^{1,2, *}$

\author{
${ }^{1}$ The University of Adelaide and Robinson Research Institute, Adelaide, Australia \\ ${ }^{2}$ South Australia Health and Medical Research Institute, Adelaide, Australia \\ ${ }^{3}$ Melbourne Brain Centre, Florey Neuroscience Institute, Parkville, Australia \\ ${ }^{4}$ Adelaide Health Technology Assessment, The University of Adelaide, Adelaide, Australia \\ *Corresponding author-paul.thomas@adelaide.edu.au
}

Running title: Behavioural phenotypes in Prrt2 mutant mice

Keywords: Prrt2, mouse model, genetic epilepsy, movement disorder, BFIE, PKD, ICCA

Submission date: $16 / 11 / 2018$

Word counts: Abstract: 156

Introduction: 474

Discussion: 870

This is the author manuscript accepted for publication and has undergone full peer review but has not been through the copyediting, typesetting, pagination and proofreading process, which may lead to differences between this version and the Version of Record. Please cite this article as doi: $10.1111 / \mathrm{gbb} .12566$

This article is protected by copyright. All rights reserved. 


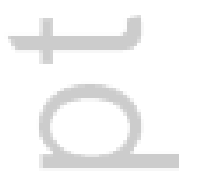

4 


\section{Abstract:}

Mutations in proline-rich transmembrane protein 2 (PRRT2) cause a range of episodic disorders that include paroxysmal kinesigenic dyskinesia (PKD) and benign familial infantile epilepsy (BFIE). Mutations are generally loss of function and include the c649dupC frameshifting mutation that is present in around $80 \%$ of affected individuals. To investigate how Prrt2 loss of function mutations cause disease, we performed a phenotypic investigation of a transgenic Prrt2 knockout (Prrt2 KO) mouse. We observed spontaneous paroxysmal episodes with behavioural features of both seizure and movement disorders, as well as unexplained deaths in KO and HET animals. KO mice showed spatial learning deficits in the Morris water maze, as well as gait abnormalities in the quantitative Digigait analysis; both of which may be representative of the more severe phenotypes experienced by homozygous patients. These findings extend the described phenotypes of Prrt2 mutant mice, further confirming their utility for in vivo investigation of the role of Prrt2 mutations in episodic diseases.

This article is protected by copyright. All rights reserved. 


\section{Introduction}

Heterozygous mutations in proline-rich transmembrane protein 2 (PRRT2) cause a range of episodic neurological disorders, most commonly benign familial infantile epilepsy (BFIE), paroxysmal kinesigenic dyskinesia (PKD) and infantile convulsions with choreoathetosis $(\text { ICCA })^{1-3}$. BFIE is an infantile epileptic seizure disorder with an onset at 3-12 months and offset around 2 years of age, without developmental impediment. In contrast, PKD is a movement disorder characterised by short bursts of hyperkinetic movement. Onset occurs in adolescence and symptoms persist into adulthood. ICCA is defined by the occurrence of BFIE and PKD in the same patient. PRRT2 mutations have also been implicated in other paroxysmal conditions, including movement disorders, hemiplegic migraine and febrile seizures $^{4-8}$. A number of patients with homozygous mutations in PRRT2 have also been reported and show increased severity of episodic disorders, along with intellectual disability 9-11. The most common disease-causing mutations result in frameshift and premature termination (for example c649dupC which occurs in almost $80 \%$ of patients) indicating a likely loss of protein function ${ }^{12}$.

Following its association with disease, investigations into the function of PRRT2 have revealed a primarily CNS-restricted protein, with a role in synaptic function ${ }^{1,13}$. PRRT2 spans 340 amino acids and contains two hydrophobic domains at the C-terminus, resulting in a membrane-bound protein of which the majority is intracellular ${ }^{1,14}$. It is expressed throughout the brain, with strongest expression in the cerebellum, hippocampus and cortex 
${ }^{1,15,16}$. PRRT2 interacts with SNARE complex proteins SNAP-25, STX1A and VAMP2, as well as $\mathrm{Ca}^{2+}$ sensors Syt1/2, all of which are important regulators of synchronous neurotransmitter release at the pre-synaptic terminal ${ }^{13,17,18}$. A study in homozygous patient-derived iPSCs revealed that PRRT2 also directly binds and negatively regulates voltage-gated $\mathrm{Na}^{+}$channels $\mathrm{Na}_{v} 1.2$ and $\mathrm{Na}_{v} 1.6$, resulting in increased $\mathrm{Na}^{+}$currents in homozygous cells ${ }^{19}$. In vitro knockdown in primary neurons results in synaptic defects; namely decreased synaptic contacts, reduced synchronous neurotransmitter release and an insensitivity to extracellular $\mathrm{Ca}^{2+18}$. A role for PRRT2 in inhibition of vesicle fusion at the synapse has also been described ${ }^{20}$. Silencing of PRRT2 in vivo causes delayed neuronal migration during development and decreased dendritic spine density ${ }^{21}$.

Recently, two groups have described mouse models lacking PRRT2 protein. Both observed paroxysmal phenotypes including spontaneous PKD-like behaviours, motor abnormalities, audiogenic movement episodes and increased seizure susceptibility/severity following stimulation with pentylentetrazol (PTZ) ${ }^{15,16}$. In this study, we extend the phenotypic investigation of the Prrt2 knockout (Prrt2 KO) mouse as a model for Prrt2-related disorders. We find that similar to patients with PRRT2 mutations, Prrt2 KO mice display a spontaneous movement disorder and seizure phenotype. We also observe premature death in both heterozygous (HET) and KO animals, along with learning deficiencies and gait abnormalities in the latter. These findings further validate this mouse model as a valuable experimental tool for investigating the underlying mechanisms of $P R R T 2$ mutations in a wide range of paroxysmal diseases.

This article is protected by copyright. All rights reserved. 


\section{Materials and methods}

Animal housing and genotyping

All animal work was conducted following approval by The University of Adelaide Animal Ethics Committee (approval numbers S-2013-201, S-2014-148, S-2016-136) in accordance with the Australian code for the care and use of animals for scientific purposes. Nine heterozygous Prrt2 ${ }^{\text {tm1a(KOMP)Wtsi }}$ founders were provided by the Australian Phenomics Network at Monash University (Melbourne, Australia) and maintained on the C57BL/6N genetic background. The colony was maintained using HET x HET crosses. For generating the behavioural testing cohort, a combination of KO x HET, WT x HET, WT x WT and KO x KO crosses were used. Animals were group-housed in an animal facility at the University of Adelaide on a 12-hour light/dark cycle and provided water and food ad libitum with meat free rat and mouse diet (Specialty Feeds, Western Australia). All testing was carried out during the light cycle. Genomic DNA for routine genotyping was obtained from tail tips or ear notches at weaning ( 3 weeks) and amplified using a multiplex PCR. The common forward primer 5'-CTGGTGCGCCTTCGAGTTGG-3' amplifies with Primer 2 5'CTGGGACCTTCTGTCTGG-3' for a WT allele or with Primer 3 5'-CAACGGGTTCTTCTGTTAGTCC$3^{\prime}$ for a transgenic allele. Amplification from inside the transgene to the $3^{\prime}$ external flanking sequence was performed using primer 5'-GTCTGAGCTCGCCATCAGTT-3' (forward) and primer 5'-GACAGCATTGAGACGTGAGC-3' (reverse).

$R T-P C R$

This article is protected by copyright. All rights reserved. 
RNA was extracted from brain tissue using Trizol reagent (Thermo Fisher Scientific). RNA was converted to cDNA using the high capacity RNA-cDNA kit (Thermo Fisher Scientific). For amplification of lacZ, forward primer 5'- TACGATGCGCCCATCTACAC-3' and reverse primer 5'-AACAACCCGTCGGATTCTCC-3' were used. For amplification of the Prrt2 ORF, forward primer 5'- CTGTCAACATTGTGGCCTTC-3' and reverse primer 5'-

GCAAAAGTGCAGGGAGAAAG-3' were used.

\section{Protein extraction and western blotting}

Brains were homogenized in extraction buffer (10mM Tris- $\mathrm{HCl}[\mathrm{pH} 7.2], 150 \mathrm{mM} \mathrm{NaCl}, 5 \mathrm{mM}$ EDTA, 1\% Triton-X-100, 1\% SDS, 1\% Deoxycholate) with Roche EDTA-free Protease Inhibitor Cocktail (Sigma Aldrich, MO, USA) and incubated at $4^{\circ} \mathrm{C}$ for 30 minutes. For chemical fractionation, Syn-PER reagent (Thermo Fisher Scientific, MA, USA) was used as per manufacturer's instructions. Lysates were separated on Invitrogen Bolt precast 4-12\% polyacrylamide gels and transferred to PVDF membrane before blotting. Membranes were blocked for 1 hour in blocking solution (5\% BSA, 5\% skim milk) at room temperature and then incubated in primary antibody/blocking solution overnight at $4^{\circ} \mathrm{C}$. Primary antibodies and their corresponding dilutions were: rabbit anti-PRRT2 (1/2000, Sigma Aldrich \#HPA014447), rabbit anti-Syt1 (1/1000, Synaptic Systems, Germany \#105103), rabbit antiSyt2 (1/1000, Synaptic Systems \#105123), rabbit anti- $\beta$-actin (1/1000, Cell Signalling, MA, USA \#4967), mouse anti-PSD95 (1/2000, Thermo Fisher Scientific \#MA1046) and rabbit antisynaptophysin (1/10000, Abcam, UK \#ab32127). Membranes were washed 3x in TBST and 
incubated for $2 \mathrm{hrs}$ at RT in secondary antibody conjugated with HRP/blocking solution at 1/400 dilution. Blots were washed and then imaged by chemiluminescence. For densitometric quantification, three biological replicates were performed and quantified using Chemidoc with Image Lab software (Biorad, CA, USA). Each band was normalized to its $\beta$-actin control, then to the relevant WT band. One-way ANOVA was performed to analyse statistical differences using GraphPad Prism 7 software (La Jolla, CA, USA).

TEM

3-4week old mice were trans-cardially perfused with ice-cold PBS followed by ice-cold $4 \%$ PFA ( $n=3$ WT, $n=3$ KO). The brains were then dissected and $1 \mathrm{~mm}$ pieces of cortex fixed in TEM fixative (4\% Sucrose, 4\% Paraformaldehyde, 1.25\% Gluteraldehyde in PBS). Tissue was postfixed for $1 \mathrm{~h}$ with osmium tetraoxide ( $2 \%$ aqueous solution) before dehydration in increasing ethanol concentrations, washing in propylene oxide and then embedding in Procure-812. Tissue was cut into ultra-thin sections $(80 \mathrm{~nm})$, stained with uranyl acetate and lead citrate and imaged on the FEI Tecnai G2 Spirit transmission electron microscope. Images were analysed using Image J software and two-tailed unpaired t-test used for statistical comparison using GraphPad Prism 7 software.

\section{Nocturnal monitoring}

Nocturnal monitoring was performed by recording with an infrared camera mounted above a Perspex-covered cage in which mice had free access to food and hydration gel. Each 
mouse was recorded for 12 hours. Video footage was reviewed for overt behavioural phenotypes.

PTZ assay

Seizure susceptibility was measured by subcutaneous injection of pentylenetetrazole (PTZ) (120 mg/kg). Prrt2 WT, HET and KO mice ( $\mathrm{n}=5$ per genotype) were observed individually in clear cylinders for drug-induced seizure phenotypes culminating in tonic-clonic seizure, at which point latency to seizure was recorded.

\section{Behavioural Testing}

Behavioural testing was carried out at the Florey Institute Behaviour Core facility (Melbourne, Australia). 8-week old female mice ( $n=10$ WT and n=14 KO) were taken through a series of behavioural tests (locomotor, rotarod, Y-maze, Digigait, Morris water maze and reversal) with a minimum of 24 hrs between each test, over a number of weeks.

Locomotor

$27.5 \mathrm{~cm}$ square clear Perspex locomotor arenas (Tru-Scan) were used for testing with $16 \mathrm{IR}$ beams on the lower sensor ring to detect floor plane movement and 16 on an elevated ring to detect rearing. Locomotive activity and rearing behaviour was recorded by the IR beam sensors over 30 minutes.

\section{Digigait}

This article is protected by copyright. All rights reserved. 
Animals were placed inside the testing chamber positioned on a clear Perspex treadmill belt. The treadmill was started at a low speed $(10 \mathrm{~cm} / \mathrm{sec})$ until the animal was comfortable walking on the treadmill. The speed was slowly increased to 15,20 or $25 \mathrm{~cm} / \mathrm{s}$ and then approximately 15 steps captured before returning the mouse to its home cage.

\section{Rotarod}

Mice were pre-trained on the rotarod (Ugo Basil, Italy) for 3, 5-minute trials. The first 2 trials were run at a constant speed of $4 \mathrm{rpm}$, and the 3rd had an accelerating speed ranging from 4 - 40 rpm over $5 \mathrm{~min}$. The testing phase was carried out the following day and involved 3 trials. Each trial consists of the accelerating setting (4-40 rpm) across the 5-minute trial. An inter-trial interval of $\sim 30$ mins was included for each animal.

\section{Spontaneous alternation using Y-maze}

Testing took place in a Y-shaped maze with three Perspex arms at a $120^{\circ}$ angle from each other. After introduction to the centre of the maze, the animal was allowed to freely explore the three arms. The number of arm entries and the number of triads were recorded in order to calculate the percentage of alternation. An entry was scored when all four limbs were within the arm.

\section{Morris Water Maze and reversal}

The $1.2 \mathrm{~m}$ diameter water maze pool was filled to a depth of $40 \mathrm{~cm}$ with 22 degree $C$ water. This leaves the $15 \mathrm{~cm}$ diameter submerged platform $1 \mathrm{~cm}$ below the water level. 
Approximately $500 \mathrm{ml}$ of non-toxic white paint was added to the water to make it opaque and hide the platform from the animal. The mouse was then placed in the pool at one of the cardinal points $(\mathrm{N}, \mathrm{E}, \mathrm{S}, \mathrm{W})$ in a randomized fashion and allowed 1 minute to find the platform. If the mouse found the platform within this time it was allowed 10 seconds on the platform before it was removed, gently towelled down and placed under a warming lamp. If the mouse did not find the platform within the 2 minutes, it was led to the platform by trailing a hand in the water in front of the mouse. It was then allowed 10 seconds on the platform before it was removed, towelled down and placed under a warming lamp. The routine was repeated 4 times per day for 6 days with an interval time between swims of 2030 minutes, until the mouse had clearly learnt the maze, signified by no significant improvement occurring after 3 consecutive days.

\section{Statistical Analysis}

Mortality data was assessed using a Fishers Exact test. Rotarod, Morris water maze and reversal data were assessed using linear mixed effects models. Where data violated distributional assumptions, it was transformed to geometric means for analysis, however arithmetic means are reported. For tests with maximal time constraints (censored data), linear mixed effects tobit models were used for statistical analysis. Locomotor data was analysed using the Wilcoxin test. Linear mixed effects models and Wilcoxin tests were completed using SAS v9.4 (SAS Institute, Cary, NC, USA). Digigait parameters were analysed with repeated measures two-way ANOVA using IBM SPSS Statistics 25. Where data violated 
sphericity, the Greenhouse-Geisser adjustment was used. Unpaired two-tailed students ttests were used for analysis of the continuous Y-maze. PTZ survival plots were analysed using the Mantel-Cox test. These analyses were performed and all graphs generated using GraphPad Prism 7 software.

This article is protected by copyright. All rights reserved. 


\section{$\underline{\text { Results }}$}

\section{Prrt2 KO mice}

Prrt2 KO mice were generated using pre-targeted ES cells purchased from KOMP (part of the International Knockout Mouse Consortium - IKMC) by ES2M/Australian Phenomics Network Facility at Monash University (Melbourne, Australia). They carry a 'knockout first' allele, encoding an engrailed (En2) splice acceptor and IRES-LacZ transgene between exon 1 and 2 of the Prrt2 locus, which is preferentially spliced resulting in Prrt2 knockout (Supplementary Figure 1a). We first established that homozygous (KO) and heterozygous (HET) Prrt2 mice expressed the IRES-LacZ transgene and that KO animals lack the Prrt2 ORF using RT-PCR (Supplementary Figure 1b). Western blot analysis on adult whole brain extract confirmed reduced PRRT2 protein in HET and an absence in Prrt2 KO animals (Supplementary Figure 1c). To confirm the location of the transgene, genomic DNA from heterozygous founders was PCR amplified using a primer outside of the transgene homology arms to within (Supplementary Figure 1d).

Chemical fractionation on adult brain samples confirmed that PRRT2 is present in the synaptosome but not the cytosolic fraction (Supplementary Figure 2a). Given that PRRT2 has been shown to interact with SYT1/2, and that knockdown of PRRT2 in primary neurons results in lower SYT1 and 2 levels ${ }^{18}$, we assessed SYT1/2 protein levels in brain lysates by western blot. No significant change in SYT1 or SYT2 was detected in the brains of Prrt2 HET or KO mice. The levels of other synapse proteins, synaptophysin and PSD95 were similarly 
unaffected by reduction or absence of PRRT2 (Supplementary Figure 2b). Transmission electron microscopy (TEM) analysis of synapses in wild type (WT) and KO cortex showed no significant difference in synapse density ( $p=0.1824$; Supplementary Figure $2 c)$ or active zone length ( $p=0.0781$; Supplementary Figure $2 d$ ).

\section{Spontaneous paroxysms and sudden unexplained death in Prrt2 mice}

PRRT2 disorders are characterised by spontaneous paroxysms, which manifest as epileptic seizures and/or a movement disorder. Consistent with these clinical features, we observed spontaneous paroxysms in Prrt2 KO mice. These events were observed during routine handling in a small proportion of Prrt2 $\mathrm{KO}$ animals, without an obvious trigger (2.83\%, Figure 1). Episodes lasted for varying lengths of time and affected individual mice at different ages (5 weeks to 8 months, summarized in Supplementary Table 1). Episodes began with dyskinesia and was in one case followed by secondary generalized seizures (which was accompanied by loss of awareness; Supplementary Video 1+2), although not confirmed by

EEG. To determine whether spontaneous paroxysms were occurring during routine housing, we performed infrared video monitoring of a small cohort during the dark cycle (the active period for mice). Overt behavioural phenotypes were not detected in either HET or KO mice (data not shown). We also assessed seizure susceptibility by performing a PTZ assay on a cohort of WT, HET and KO adult mice ( $n=5$ per genotype) but observed no significant difference in latency to tonic-clonic seizure (Supplementary Figure 3).

This article is protected by copyright. All rights reserved. 
Interestingly, Prrt2 KO mice also displayed a marked survival defect with $8.49 \%$ of KO found dead in their cages, at different ages, without any clear cause of death (Figure 1, Supplementary Figure 4). A Fishers Exact test revealed a significant difference between groups, with KO mice showing higher mortality than WT and HET ( $p=0.0003)$. Both the spontaneous paroxysms and unexplained deaths were not observed in WT animals from the same colony, confirming Prrt2 mutation as the cause of these phenotypes.

\section{Gait alteration in Prrt2 KO mice}

To assess motor activity and coordination in Prrt2 KO mice, we performed locomotor, digigait and rotarod behavioural testing. Given that phenotypic severity is greater in PRRT2 homozygous patients, we compared Prrt2 KO $(n=14)$ and WT $(n=10)$ mice. Locomotor analysis showed no overall difference in movement or activity between Prrt2 KO and WT (Supplementary Figure 5a). No significant difference was observed between groups of animals on the Rotarod test, indicating that Prrt2 mice do not suffer from coordination issues (Supplementary Figure 5b).

Quantitative comparison of gait was performed using digigait analysis across three speeds $(15,20$ and $25 \mathrm{~cm} / \mathrm{s})$. Interestingly, significant differences were detected between WT and KO mice across most aspects of gait including stride, swing, stance and brake. Stride length was significantly lower, whilst number of steps and step frequency were significantly higher in $\mathrm{KO}$ animals (Figure 2, Table 1). To further define these differences, post hoc Sidak multiple comparison tests were performed. This analysis revealed significant differences between

This article is protected by copyright. All rights reserved. 
WT and $\mathrm{KO}$ mice at $15 \mathrm{~cm} / \mathrm{s}$ and to a large extent at $20 \mathrm{~cm} / \mathrm{s}$, but not at $25 \mathrm{~cm} / \mathrm{s}$

(Supplementary Table 2). To rule out the possibility that animal size was the cause of the differences, we compared animal length between WT and KO animals and saw no significant difference $(p=0.3671$, Supplementary Figure $5 c)$. Altogether, digigait analysis on Prrt2 KO mice show a comprehensive series of abnormalities across many components of gait.

\section{Prrt2 KO mice display learning deficiencies}

Given that intellectual disability is observed in homozygous patients, we investigated the cognitive abilities of KO mice. When tested on the continuous Y-maze, there was no significant difference between Prrt2 KO and WT animals in percentage alternation between arms ( $p=0.1992 ;$ Supplementary Figure 6a).

In the Morris Water Maze, Prrt2 KO mice displayed significantly longer latency to platform than WT (Chisquare (1 degree of freedom $)=5.99, p=0.014$, Figure 3a). Post hoc analysis confirmed an overall genotype difference that was independent of trial day. When we measured velocity in Morris Water Maze, no differences were observed between KO and WT, indicating that differences observed were due to cognitive deficits rather than reduced or slower swimming ability (Supplementary Figure 6b,c). When spatial memory was tested during the probe trial, there was no significant difference in target quadrant time between genotypes ( $p=0.083$, Figure $3 b)$. Repeating the Morris Water maze with reversal of the platform resulted in no differences between genotypes in latency to platform across 5 days nor in target quadrant time in a reversal probe test $(p=0.906$ or 0.693 respectively, Figure 
$3 c, d)$. Together these data suggest learning defects in Prrt2 KO mice, in the absence of memory deficits.

This article is protected by copyright. All rights reserved. 


\section{Discussion}

Heterozygous loss of function mutations in $P R R T 2$ cause a broad spectrum of episodic diseases, most frequently BFIE and PKD. Analysis of gene function using knockdown techniques has revealed a synaptic function for the protein, as well as a possible role in neuronal development ${ }^{18,21}$.

In order to investigate the impact of Prrt2 mutations in vivo we undertook phenotypic analysis of a Prrt2 KO mouse. Consistent with a recent report on the same transgenic model, we observed no detectable protein or transcript in the brains of $\mathrm{KO}$ animals, with reduced levels in heterozygous mutants ${ }^{15}$. PRRT2 localised to the synapse as previously reported $13,16,18,21$, though its reduction or knockout did not change levels of SYT $1 / 2$ in vivo as observed in PRRT2 knockdown neurons ${ }^{18}$. When we assessed cortical synaptic density by TEM, we saw no significant differences between WT and Prrt2 KO brains. The same observation was recently described in the cerebellum of a Prrt $2^{\text {STOP }}$ mouse that models the recurrent c649dupC mutation in patients ${ }^{16}$. Interestingly, Michetti et al reported decreased synaptic contacts in the dentate gyrus (DG) but not in two regions of the cerebellum. Similarly, they observed that mRNA expression levels of most synaptic markers were not altered in the cortex of Prrt2 HET or KO animals ${ }^{15}$. This suggests that although Prrt2 is expressed broadly throughout the brain, it may be playing a different role in the DG compared to other brain regions.

This article is protected by copyright. All rights reserved. 
We describe a series of behavioural phenotypes in Prrt2 KO mice that model clinical phenotypes observed in humans. Most notably, spontaneous paroxysms were detected in Prrt2 KO mice, albeit at low penetrance. A similar phenotype was described by Tan et al, with low frequency spontaneous dyskinetic attacks observed in Prrt2 ${ }^{\text {STOP }}$ mice ${ }^{16}$. Consistent with a previous analysis of the Prrt2 KO model, when we probed the seizure susceptibility by PTZ injection, we observed no difference in latency to tonic-clonic seizure for both Prrt2 HET and KO mice, though the previous study observed more severe seizures once induced ${ }^{15}$. In contrast, increased seizure susceptibility was observed in Prrt2 ${ }^{\text {STOP }}$ mice using both a PTZ and febrile seizure assay ${ }^{16}$. The models are maintained on different C57BL/ 6 substrains, which have genotypic and phenotypic differences that may impact on performance in behavioural tests ${ }^{22}$. We also identified unexplained death in a subset of Prrt2 mutant (HET and KO) animals (Figure 1). Though not a common feature of $P R R T 2$ mutations in humans, a heterozygous patient with probable sudden unexpected death in epilepsy (SUDEP) has been reported ${ }^{23}$. Other murine models of genetic epilepsies have also been identified with a premature death phenotype, although it is not clear if the primary cause of death is related 24,25 .

Whilst most PRRT2-related disorders are paroxysmal in nature, homozygous patients experience increased severity of disease, along with additional intellectual and learning disabilities ${ }^{10,11}$. Consistent with these phenotypes, we observed learning defects in Prrt2 KO mice when testing their spatial learning in the Morris Water Maze (Figure 3a). However, working memory and memory plasticity were unaffected as evidenced by the probe test and 
platform reversal ${ }^{26}$. Rotarod and locomotor tests did not reveal a motor or coordination phenotype for the Prrt2 KO mice in our study, despite the coordination differences described in Prrt2 ${ }^{\text {STOP }}$ mice ${ }^{16}$. Prrt2 KO mice also displayed gait abnormalities when assessed on the Digigait software. These abnormalities span many fundamental gait parameters and manifested when travelling at the slowest treadmill speed $(15 \mathrm{~cm} / \mathrm{s})$ during digigait analysis (Table 1, Supplementary Table 2). Interestingly, these differences were not as pronounced at the faster speed $(20 \mathrm{~cm} / \mathrm{s})$ and were virtually absent at the fastest speed $(25 \mathrm{~cm} / \mathrm{s})$. Differences in gait can be speed-dependent, with faster speeds (akin to running) masking phenotypes observed at slower (walking) speeds (unpublished observations, $\mathrm{T}$. Featherby). Previously reported footprint analysis of Prrt2 mice showed no differences between genotypes, however many aspects of gait that differed here were not tested and mice were not controlled for speed ${ }^{15,16}$. Supplementary Table 3 summarises the behavioural phenotypes described in our study and previously published Prrt2 mouse model analysis. Movement disorders experienced by patients with PRRT2 mutations are typically episodic in nature and do not extend to sustained chorea or ataxia, although there is some evidence of motor deficits and coordination difficulties in homozygous patients ${ }^{10,11}$. Therefore, the finding of overall gait differences is somewhat unexpected. We cannot rule out the possibility that subtle movement episodes were experienced by a subset of KO animals during testing, resulting in the overall gait deficit attributed to the group, however given the lack of significant differences at the fastest speed, this seems unlikely. It remains to be seen how Prrt2 HET animals perform in these same behavioural experiments. 
However, we expect that learning and gait phenotypes will not be present, given that Prrt2 heterozygosity in humans most commonly results in epileptic or motor paroxysms with an absence of other phenotypes.

Together, our phenotypic investigation of the Prrt2 KO mouse revealed a paroxysmal phenotype in $\mathrm{KO}$, along with premature death affecting $\mathrm{HET}$ and $\mathrm{KO}$ animals. Wide-ranging gait abnormalities and learning deficits were also observed in KO mice. These data support Prrt2 KO mice as a useful model for investigating Prrt2 function and elucidation of disease mechanisms underlying the PRRT2-related disease spectrum.

\section{Acknowledgments}

We would like to acknowledge the Adelaide Microscopy facility, particularly Ruth Williams, for assistance with and provision of the transmission electron microscope. We also acknowledge the facilities, and the scientific and technical assistance of ES2M, Monash University for generating the Prrt2 KO mouse model. ES2M is supported by the Australian Phenomics Network (APN). The APN is supported by the Australian Government through the National Collaborative Research Infrastructure Strategy (NCRIS) program. We would like to thank Kay Richards and Chris Reid for expert interpretations of the behavioural manifestations of the paroxysmal events. This work was supported by funding from the National Health and Medical Research Council and Channel 7 Children's Research Foundation.

This article is protected by copyright. All rights reserved. 


\section{References}

1. Chen $\mathrm{WJ}$, Lin $\mathrm{Y}$, Xiong $Z Q$, et al. Exome sequencing identifies truncating mutations in PRRT2 that cause paroxysmal kinesigenic dyskinesia. Nat Genet. 2011;43(12):12521255.

2. Wang JL, Cao L, Li XH, et al. Identification of PRRT2 as the causative gene of paroxysmal kinesigenic dyskinesias. Brain. 2011;134(Pt 12):3493-3501.

3. Heron SE, Grinton BE, Kivity S, et al. PRRT2 mutations cause benign familial infantile epilepsy and infantile convulsions with choreoathetosis syndrome. Am J Hum Genet. 2012;90(1):152-160.

4. Liu Q, Qi Z, Wan XH, et al. Mutations in PRRT2 result in paroxysmal dyskinesias with marked variability in clinical expression. J Med Genet. 2012;49(2):79-82.

5. Cloarec R, Bruneau N, Rudolf G, et al. PRRT2 links infantile convulsions and paroxysmal dyskinesia with migraine. Neurology. 2012;79(21):2097-2103.

6. Gardiner AR, Bhatia KP, Stamelou M, et al. PRRT2 gene mutations: from paroxysmal dyskinesia to episodic ataxia and hemiplegic migraine. Neurology. 2012;79(21):21152121.

7. Marini C, Conti V, Mei D, et al. PRRT2 mutations in familial infantile seizures, paroxysmal dyskinesia, and hemiplegic migraine. Neurology. 2012;79(21):2109-2114.

8. Scheffer IE, Grinton BE, Heron SE, et al. PRRT2 phenotypic spectrum includes sporadic and fever-related infantile seizures. Neurology. 2012;79(21):2104-2108.

9. Ebrahimi-Fakhari D, Saffari A, Westenberger A, Klein C. The evolving spectrum of PRRT2-associated paroxysmal diseases. Brain. 2015;138(Pt 12):3476-3495.

10. Labate A, Tarantino P, Viri M, et al. Homozygous c.649dupC mutation in PRRT2 worsens the BFIS/PKD phenotype with mental retardation, episodic ataxia, and absences. Epilepsia. 2012;53(12):e196-199.

11. Delcourt M, Riant F, Mancini J, et al. Severe phenotypic spectrum of biallelic mutations in PRRT2 gene. J Neurol Neurosurg Psychiatry. 2015;86(7):782-785.

12. Heron SE, Dibbens LM. Role of PRRT2 in common paroxysmal neurological disorders: a gene with remarkable pleiotropy. J Med Genet. 2013;50(3):133-139.

13. Lee HY, Huang $\mathrm{Y}$, Bruneau N, et al. Mutations in the gene PRRT2 cause paroxysmal kinesigenic dyskinesia with infantile convulsions. Cell Rep. 2012;1(1):2-12.

This article is protected by copyright. All rights reserved. 
14. Rossi P, Sterlini B, Castroflorio E, et al. A Novel Topology of Proline-rich Transmembrane Protein 2 (PRRT2): hints for an intracellular function at the synapse. J Biol Chem. 2016;291(12):6111-6123.

15. Michetti C, Castroflorio E, Marchionni I, et al. The PRRT2 knockout mouse recapitulates the neurological diseases associated with PRRT2 mutations. Neurobiol Dis. 2017;99:66-83.

16. Tan GH, Liu YY, Wang L, et al. PRRT2 deficiency induces paroxysmal kinesigenic dyskinesia by regulating synaptic transmission in cerebellum. Cell Res. 2017.

17. Valtorta F, Benfenati F, Zara F, Meldolesi J. PRRT2: from Paroxysmal Disorders to Regulation of Synaptic Function. Trends Neurosci. 2016;39(10):668-679.

18. Valente $\mathrm{P}$, Castroflorio $\mathrm{E}$, Rossi $\mathrm{P}$, et al. PRRT2 Is a Key Component of the $\mathrm{Ca}(2+)$ Dependent Neurotransmitter Release Machinery. Cell Rep. 2016;15(1):117-131.

19. Fruscione $F$, Valente $P$, Sterlini $B$, et al. PRRT2 controls neuronal excitability by negatively modulating Na+ channel 1.2/1.6 activity. Brain. 2018;141(4):1000-1016.

20. Coleman J, Jouannot O, Ramakrishnan SK, et al. PRRT2 Regulates Synaptic Fusion by Directly Modulating SNARE Complex Assembly. Cell Rep. 2018;22(3):820-831.

21. Liu YT, Nian FS, Chou WJ, et al. PRRT2 mutations lead to neuronal dysfunction and neurodevelopmental defects. Oncotarget. 2016;7(26):39184-39196.

22. Simon MM, Greenaway S, White JK, et al. A comparative phenotypic and genomic analysis of C57BL/6J and C57BL/6N mouse strains. Genome Biol. 2013;14(7):R82.

23. Labate A, Tarantino P, Palamara G, et al. Mutations in PRRT2 result in familial infantile seizures with heterogeneous phenotypes including febrile convulsions and probable SUDEP. Epilepsy Res. 2013;104(3):280-284.

24. Massey CA, Sowers LP, Dlouhy BJ, Richerson GB. Mechanisms of sudden unexpected death in epilepsy: the pathway to prevention. Nat Rev Neurol. 2014;10(5):271-282.

25. Palmer EE, Jarrett KE, Sachdev RK, et al. Neuronal deficiency of ARV1 causes an autosomal recessive epileptic encephalopathy. Hum Mol Genet. 2016;25(14):30423054.

26. Tsetsenis T, Younts TJ, Chiu CQ, Kaeser PS, Castillo PE, Sudhof TC. Rab3B protein is required for long-term depression of hippocampal inhibitory synapses and for normal reversal learning. Proc Natl Acad Sci U S A. 2011;108(34):14300-14305.

This article is protected by copyright. All rights reserved. 


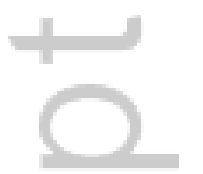

4 


\section{Figure Legends}

Figure 1: Proportions of Prrt2 HET and $\mathrm{KO}$ animals that experienced spontaneous death and seizures. Animals that were found dead are represented by red shading, those that experienced spontaneous paroxysms are in green and unaffected mice are indicated by blue. No adverse events were observed in WT animals from the Prrt2 colony.

Figure 2: Gait deficiencies in Prrt2 KO mice. (a,b) Stride time across three speeds $(15,20$ \& $25 \mathrm{~cm} / \mathrm{s})$ was significantly lower in KO compared to WT in both fore $(\mathrm{p}=0.0016)$ and hind limbs ( $p=0.0004)$. ( $c, d)$ Prrt2 KO mice showed shorter stride length than WT in both fore $(p=0.0051)$ and hind limbs ( $p=0.0019)$ across the three speeds. Differences between genotypes were analysed with repeated measures two-way ANOVA. Error bars indicate mean \pm SEM. $n=20$ WT (10 mice, measurements for $R$ and $L$ paws) and $n=28$ KO (14 mice, measurements for $\mathrm{R}$ and $\mathrm{L}$ paws). ${ }^{* *}: \mathrm{p}<0.01,{ }^{* * *}: \mathrm{p}<0.001$.

Figure 3: Learning disabilities in Prrt2 KO mice (a) Prrt2 KO mice show significantly longer latency to platform in the Morris Water Maze (MWM) training days ( $p=0.014)$. This difference was independent of day. (b) Percentage of time spent in each quadrant during the probe trial with the platform removed. The platform had previously been in the NE quadrant during training days. Differences between WT and KO were not significant $(p=0.083)$. (c) The platform was moved to the SW quadrant for the MWM reversal. Latency to find platform across the 5 reversals days was not significant between WT and KO $(p=0.906)$. ( $d)$ Percentage time spent in each quadrant during the reversal probe trial. Differences between WT and KO were not significant ( $p=0.693$ ). MWM and reversal training day analyses were performed using a linear mixed effects tobit model. Probe data was analysed using a linear mixed effects model. Data expressed as mean \pm SEM. $n=40$ WT (10 mice, 4 trials each) and $n=56 \mathrm{KO}$ (14 mice, 4 trials each). Ns: not significant, *: $p<0.05$.

Table 1: Genotype comparison across gait parameters from Digigait testing showing main effect for genotype test statistic and $p$-value using repeated measures two-way ANOVA. Significant $p$-values highlighted in bold. $n=20$ WT (10 mice, $R$ and L paw measurements), $\mathrm{n}=28 \mathrm{KO}$ (14 mice, $\mathrm{R}$ and $\mathrm{L}$ paw measurements).

This article is protected by copyright. All rights reserved. 

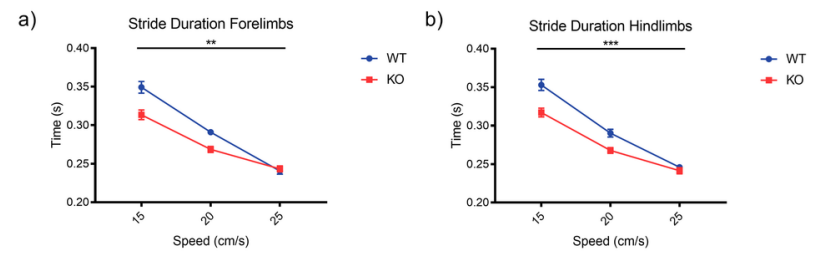

d)

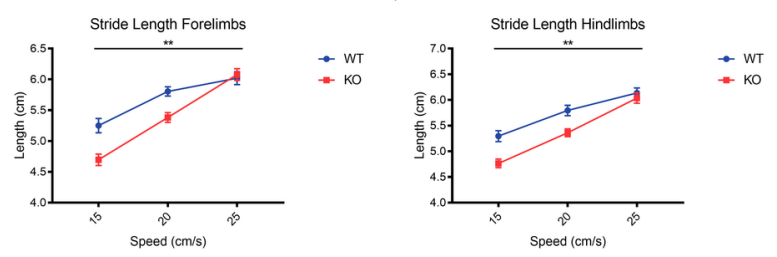

GBB_12566_RobertsonFigure 2.tif

This article is protected by copyright. All rights reserved. 


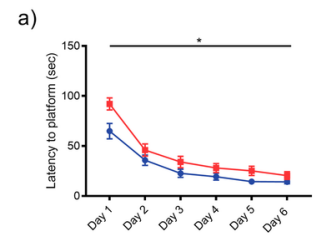

c)

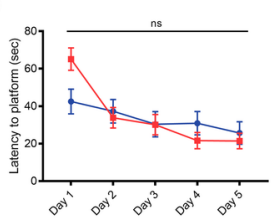

b)

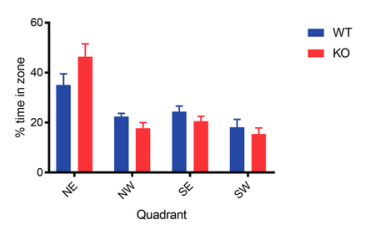

d)

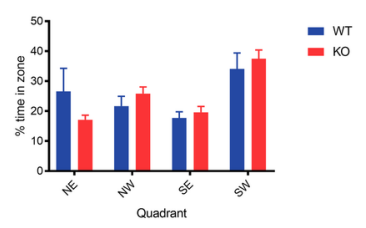

GBB_12566_RobertsonFigure 3.tif 


\begin{tabular}{|c|c|c|c|c|c|c|c|c|c|c|c|c|c|c|c|c|c|c|}
\hline \multirow{2}{*}{$\begin{array}{c}\begin{array}{c}\text { Digigait } \\
\text { Parameter }\end{array} \\
\text { Limb }\end{array}$} & \multicolumn{2}{|c|}{$\begin{array}{c}\text { Stride } \\
\text { duration }\end{array}$} & \multicolumn{2}{|c|}{ Swing } & \multicolumn{2}{|c|}{ Stance } & \multicolumn{2}{|c|}{ Brake } & \multicolumn{2}{|c|}{ Propel } & \multicolumn{2}{|c|}{$\begin{array}{c}\text { Stride } \\
\text { Frequency }\end{array}$} & \multicolumn{2}{|c|}{$\begin{array}{c}\begin{array}{c}\text { Number of } \\
\text { Steps }\end{array} \\
\end{array}$} & \multicolumn{2}{|c|}{$\begin{array}{l}\text { Stride } \\
\text { length } \\
\end{array}$} & \multicolumn{2}{|c|}{$\begin{array}{l}\text { Stance } \\
\text { Width }\end{array}$} \\
\hline & Fore & Hind & Fore & Hind & Fore & Hind & Fore & Hind & Fore & Hind & Fore & Hind & Fore & Hind & Fore & Hind & Fore & Hind \\
\hline $\begin{array}{c}\text { Genotype } \\
\text { main } \\
\text { effect }\end{array}$ & $\begin{array}{c}\mathrm{F} 1,46= \\
11.29, \\
\mathrm{p}= \\
\mathbf{0 . 0 0 1 6} \\
* *\end{array}$ & $\begin{array}{c}\mathrm{F} 1,46= \\
14.48, \\
\mathbf{p}= \\
\mathbf{0 . 0 0 0 4} \\
* * *\end{array}$ & $\begin{array}{c}\mathrm{F} 1,46= \\
1.338 \\
\mathrm{p}= \\
0.2533 \\
\mathrm{~ns}\end{array}$ & $\begin{array}{c}\mathrm{F} 1,46= \\
5.731, \\
\mathbf{p}= \\
\mathbf{0 . 0 2 0 8} \\
*\end{array}$ & $\begin{array}{c}\mathrm{F} 1,46= \\
18.01, \\
\mathrm{p}= \\
\mathbf{0 . 0 0 0 1} \\
* * *\end{array}$ & $\begin{array}{c}\mathrm{F} 1,46= \\
13.38, \\
\mathbf{p}= \\
\mathbf{0 . 0 0 0 7} \\
* * *\end{array}$ & $\begin{array}{c}\mathrm{F} 1,46= \\
6.529, \\
p= \\
0.0140 \\
*\end{array}$ & $\begin{array}{c}\mathrm{F} 1,46= \\
11.36, \\
\mathrm{p}= \\
\mathbf{0 . 0 0 1 5} \\
* *\end{array}$ & $\begin{array}{c}\mathrm{F} 1,46= \\
1.712 \\
\mathrm{p}= \\
0.1972 \\
\mathrm{~ns}\end{array}$ & $\begin{array}{c}F 1,46= \\
2.515 \\
p= \\
0.1196 \\
\text { ns }\end{array}$ & $\begin{array}{c}\mathrm{F} 1,46= \\
7.9 \\
\mathrm{p}= \\
\mathbf{0 . 0 0 7 2} \\
* *\end{array}$ & $\begin{array}{c}\mathrm{F} 1,46= \\
11.4 \\
\mathbf{p}= \\
\mathbf{0 . 0 0 1 5} \\
* *\end{array}$ & $\begin{array}{c}F 1,46= \\
2.623, \\
p= \\
0.1121 \\
n s\end{array}$ & $\begin{array}{c}\mathrm{F} 1,46= \\
5.078 \\
p= \\
0.0290 \\
*\end{array}$ & $\begin{array}{c}\mathrm{F} 1,46= \\
8.67 \\
\mathrm{p}= \\
0.0051 \\
* *\end{array}$ & $\begin{array}{c}\mathrm{F} 1,46= \\
10.91, \\
\mathrm{p}= \\
0.0019 \\
* *\end{array}$ & $\begin{array}{c}\mathrm{F} 1,22= \\
8.067, \\
\mathrm{p}= \\
0.0095 \\
* *\end{array}$ & $\begin{array}{c}\mathrm{F} 1,22= \\
0.137, \\
\mathrm{p}= \\
0.7152 \\
\mathrm{~ns}\end{array}$ \\
\hline
\end{tabular}

Key- ns: not significant, ${ }^{*}: p<0.05,{ }^{* *}: p<0.01,{ }^{* * *}: p<0.001,{ }^{* * * *}: p<0.0001$. 


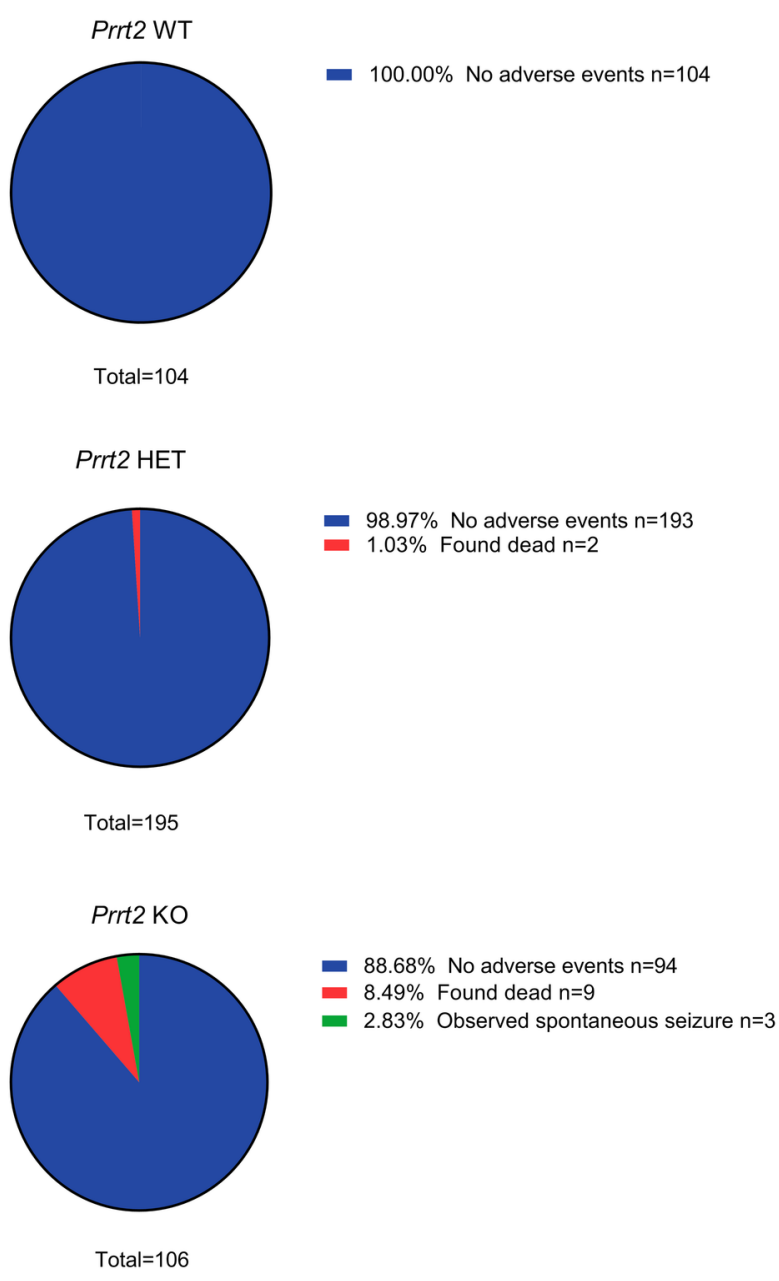

GBB_12566_updatedRobertsonFigure 1.tif

This article is protected by copyright. All rights reserved. 


\section{University Library}

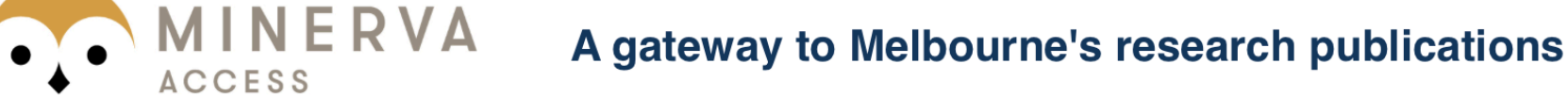

Minerva Access is the Institutional Repository of The University of Melbourne

Author/s:

Robertson, L;Featherby, T;Howell, S;Hughes, J;Thomas, P

Title:

Paroxysmal and cognitive phenotypes in Prrt2 mutant mice.

Date:

2019-06

Citation:

Robertson, L., Featherby, T., Howell, S., Hughes, J. \& Thomas, P. (2019). Paroxysmal and cognitive phenotypes in Prrt2 mutant mice.. Genes Brain Behav, 18 (5), pp.e12566-. https:// doi.org/10.1111/gbb.12566.

Persistent Link:

http://hdl.handle.net/11343/285668 